\title{
Subelliptic $p$-harmonic maps into spheres and the ghost of Hardy spaces
}

\author{
Piotr Hajłasz, Pawel Strzelecki * \\ Institute of Mathematics, Warsaw University, ul. Banacha 2, 02-097 Warszawa, Poland \\ (e-mail: hajlasz@mimuw.edu.pl / pawelst@mimuw.edu.pl)
}

Received: 27 November 1997

Mathematics Subject Classification (1991): 35B65, 35J70

\section{Introduction}

The story begins with the paper of Müller, [59], who - for the sake of an application to nonlinear elasticity - proved that if the Jacobian determinant $J_{u}$ of a Sobolev map $u \in W_{\text {loc }}^{1, n}\left(\mathbb{R}^{n}, \mathbb{R}^{n}\right)$ is nonnegative, then it belongs locally to $L \log L$. The result is quite intriguing, since a priori Hölder inequality implies only that $J_{u} \in L^{1}$ and one does not suspect any higher integrability.

If one does not assume that $J_{u}$ is nonnegative, then, as Coifman, Lions, Meyer and Semmes [14] have proved, $J_{u}$ belongs to the local Hardy space $\mathscr{H}_{\mathrm{loc}}^{1}$. Since a nonnegative function belongs to the local Hardy space if and only if it belongs locally to $L \log L$, the result of Coifman, Lions, Meyer and Semmes generalizes that of Müller.

In fact, Coifman, Lions, Meyer and Semmes proved more. Namely, for $1<$ $p<\infty$, the scalar product of a divergence free vector field $E \in L^{p}\left(\mathbb{R}^{n}, \mathbb{R}^{n}\right)$ and a curl free vector field $B \in L^{p /(p-1)}\left(\mathbb{R}^{n}, \mathbb{R}^{n}\right)$ belongs to the Hardy space $\mathscr{\mathscr { C }}{ }^{1}$. A priori, Hölder inequality implies integrability of this expression only. Note that the Jacobian is of this form. The discovery that many algebraic expressions involving partial derivatives belong to the Hardy space turned out to be important and widely applicable in nonlinear partial differential equations. This was first shown by Hélein, [40], [41], [42]. Let us describe briefly his result.

Consider maps $u: B^{n} \rightarrow S^{m}$ from the $n$-dimensional ball to the $m$ dimensional sphere such that the $p$-energy of $u$, given by the functional

\footnotetext{
* Both authors were supported by KBN grant no. 2-PO3A-034-08. The research was completed during the stay of the first author in the Max-Planck Institute for Mathematics in the Sciences in Leipzig in 1997. He wishes to thank the Institute for the support and the hospitality.
} 


$$
E_{p}(u)=\int_{B^{n}}|\nabla u|^{p}=\int_{B^{n}}\left(\sum_{i, j=1}^{m+1}\left(\frac{\partial u_{i}}{\partial x_{j}}\right)^{2}\right)^{p / 2},
$$

is finite. Here $S^{m}$ is the unit sphere in $\mathbb{R}^{m+1}$, so that $u=\left(u_{1}, \ldots, u_{m+1}\right)$ is a map into $\mathbb{R}^{m+1}$ satisfying $\sum_{j} u_{j}^{2}=1$ a.e. It is natural to assume that $u$ belongs to the Sobolev space $W^{1, p}$, in the sense that all the coordinate functions $u_{j}$ belong to $W^{1, p}\left(B^{n}\right)$.

Critical points with respect to variations in the range (see the definition in Sect. 3) of the functional $E_{p}$ satisfy the Euler-Lagrange system

$$
-\operatorname{div}\left(|\nabla u|^{p-2} \nabla u\right)=u|\nabla u|^{p} .
$$

This is a system of $m+1$ equations (since $u$ takes values in $\mathbb{R}^{m+1}$ ) which, for $n=p=2$, reduces to

$$
-\Delta u=u|\nabla u|^{2} .
$$

Hélein proved that in this particular situation $u$ is smooth. (It was known that continuity implies smoothness of $u$ so it was enough to prove that $u$ is continuous.) Using the constraint condition $\sum_{i} u_{i}^{2}=1$, Hélein could apply the theorem of Coifman, Lions, Meyer and Semmes to check that the right hand side of (2) belongs in fact to the Hardy space; hence, (2) could be rewritten in an equivalent form $\Delta u=h$, with $h \in \mathscr{\mathscr { C }}{ }^{1}$. Now, using the fact that convolution with the fundamental solution $I_{2}$ of the Laplacian is a bounded operator from $\mathscr{\mathscr { H }}{ }^{1}$ to $\mathrm{W}^{2,1}$, he concluded that $u$ is of class $W^{2,1}$ and hence continuous. The boundedness of the convolution operator follows from the theory of Hardy spaces. Indeed, computing the second order derivatives of $I_{2} * h$ we obtain Riesz operators which, due to the theory of singular integrals, are bounded on $\mathscr{H} \mathscr{C}^{1}$.

Later Fuchs [31], and Strzelecki [67], generalized Hélein's result to the case $n=p \geq 2$. We follow the argument of [67]. Here again, applying the result of [14], one obtains an equation of the form $\operatorname{div}\left(|\nabla u|^{p-2} \nabla u\right)=h$ with $h \in \mathscr{H}^{1}$. When $p>2$, the convolution with the fundamental solution is not available any more. Instead of that Strzelecki employed the duality of $\mathscr{H}^{1}$ and BMO. Namely, $W^{1, p}\left(B^{p}\right) \subset$ BMO, so, for $\eta$ supported on a ball $B$, test functions of the form $\eta\left(u-u_{B}\right)$ can be integrated against $h=u|\nabla u|^{p}$. This leads to Caccioppoli type estimates which in turn imply Hölder continuity of $u$.

There are, of course, many other papers which employ similar ideas. To mention just a few of them (we do not claim that the list below is complete), let us mention here Evans [18], Evans and Müller [19], Bethuel [1], Bethuel and Ghidaglia [2], Mou and Yang [58], Müller and Šverák [60], Semmes [65], Strzelecki [68], Takeuchi [70], and Toro and Wang [71]. All these authors use Hardy spaces in order to obtain the regularity of solutions to some nonlinear partial differential equations.

The aim of this paper is to seek for counterparts of those results in situations where Hardy spaces are not available but a weak counterpart of $\mathscr{H}^{1}-\mathrm{BMO}$ duality still holds true. In fact, in the papers mentioned above (at least in some of them), instead of using the theory of Hardy spaces, it suffices to apply a 
weaker inequality which is available in a much more general setting. Already Chanillo [9] has shown how to bypass the $\mathscr{H} \mathscr{C}^{1}-$ BMO duality in the Euclidean case of stationary harmonic maps. However, his proof heavily relies on harmonic analysis in $\mathbb{R}^{n}$, and does not seem to be applicable in our much more general situation. In the simplified approach to duality inequality we use some ideas of Semmes, [65].

We deal with a family of vector fields $X_{1}, \ldots, X_{k}$ in $\mathbb{R}^{n}$ satisfying the socalled Hörmander's condition (see Sect. 2 for the definition and more details). Let $\Omega \subset \mathbb{R}^{n}$ be a bounded domain. Any map which is stationary with respect to variations in the range for the energy functional

$$
E_{p}(u)=\int_{\Omega}|X u|^{p},
$$

defined on the class of maps into the Euclidean sphere $S^{m}$, satisfies a system of nonlinear subelliptic equations,

$$
-\sum_{j=1}^{k} X_{j}^{*}\left(|X u|^{p-2} X_{j} u\right)=u|X u|^{p}
$$

and the constraint $\sum_{j} u_{j}^{2}=1$. Such maps will be called subelliptic p-harmonic. The idea of generalizing the concept of $p$-harmonic maps to the setting of Hörmander vector fields, or even more general setting of metric spaces is not new, see Jost [46], [47], and Jost and $\mathrm{Xu}$ [48].

With a system of vector fields satisfying Hörmander's conditions one can associate the so called homogeneous dimension (see Sect. 2). This dimension is, in general, greater than Euclidean dimension but, roughly speaking, with respect to the vector fields $X_{j}$ it behaves like the classical Euclidean dimension for the standard vector fields $\partial_{1}, \ldots, \partial_{n}$.

We prove that when $p$ is equal to the homogeneous dimension associated to a given system of vector fields, the solutions to the above nonlinear system with constraints are Hölder continuous (Theorem 3.1).

Our motivation is twofold. First, we show that one does not have to use the theory of Hardy spaces; instead, a weaker version of duality inequality can be applied. We prove in Lemma 3.2 that such a duality inequality holds true also for general Hörmander vector fields (when there is no theory of Hardy spaces). The second goal is to contribute to the theory of nonlinear subelliptic equations during last decade, an area of intensive research; see, e.g., Buckley, Koskela and Lu [4], Capogna, Danielli and Garofalo [5], [7], Citti [11], Citti and Di Fazio [12], Citti, Garofalo and Lanconelli [13], Danielli, Garofalo and Nhieu [17], Franchi and Lanconelli [26], Franchi, Gutiérrez and Wheeden [25], Garofalo and Lanconelli [32], Garofalo and Nhieu [34], Hajkasz and Koskela [38], [39], Jerison and Lee [44], [45] Jost and Xu [48], Lu [52], [53], [54], Vodop'yanov, [74], Vodop'yanov and Chernikov [75], Vodop'yanov and Markina [76], Xu [77], [78], and their references. (We did not mention here any papers concerned with the linear theory of subelliptic equations.) 
We develop a technique which allows one to extend the above mentioned results of Hélein, Evans, Bethuel and others to the setting of vector fields. Only one particular question is treated in this paper, but similar methods can be used to deal with other problems.

Our notation is fairly standard. The average value will be denoted by

$$
u_{E}=f_{E} u d \mu=\frac{1}{\mu(E)} \int_{E} u d \mu
$$

The Lebesgue measure of the set $A$ will be denoted by $|A|$. Balls will be denoted by $B$. The ball concentric with $B$ and with radius $\sigma$ times that of $B$ will be denoted by $\sigma B$. By $C$ we will denote a general constant; it can change even in a single string of estimates. We say that two quantities are comparable, and write $A \approx B$, if there is a constant $c \geq 1$ such that $A / c \leq B \leq c A$

Outline of the paper. In the next section, we fix the rest of notation and gather all necessary "subelliptic technicalities". The precise statement and proof of our regularity result for subelliptic $p$-harmonic maps is given in Sect. 3.

\section{Auxiliary results}

The aim of the section is to recall some definitions and collect the results which will be used in the sequel. The reader who is familiar with the theory of vector fields satisfying Hörmander's condition can jump directly to the statement of the main theorem in Sect. 3, and then, during the lecture of the proof, she or he may consult previous subsections.

\subsection{The Hörmander condition}

Let the vector fields $X_{1}, X_{2}, \ldots, X_{k}$ be defined in $\mathbb{R}^{n}$, real valued, and $C^{\infty}$ smooth. We say that these vector fields satisfy Hörmander's condition provided there exists an integer $m$ such that the family of commutators of $X_{1}, X_{2}, \ldots, X_{k}$ up to length $m$ i.e., the family of vector fields

$$
X_{1}, \ldots, X_{k}, \quad\left[X_{i_{1}}, X_{i_{2}}\right], \quad \ldots, \quad\left[X_{i_{1}},\left[X_{i_{2}},\left[\ldots, X_{i_{m}}\right]\right] \ldots\right], \quad i_{j}=1,2, \ldots, k,
$$

spans the tangent space $\mathbb{R}^{n}$ at every point of $\mathbb{R}^{n}$.

Given any real valued Lipschitz continuous function $u \in \operatorname{Lip}\left(\mathbb{R}^{n}\right)$, we define

$$
X_{j} u(x)=\left\langle X_{j}(x), \nabla u(x)\right\rangle, \quad j=1,2, \ldots, k .
$$

The vector with components $X_{i} u$ is denoted by $X u$; its length is given by

$$
|X u(x)|^{2}=\sum_{j=1}^{k}\left|X_{j} u(x)\right|^{2} .
$$

By $X_{j}^{*}$ we will denote formal adjoint of $X_{j}$ on $L^{2}$, i.e., 


$$
\int_{\mathbb{R}^{n}}\left(X_{j}^{*} u\right) v d x=-\int_{\mathbb{R}^{n}} u X_{j} v d x \quad \text { for all } u, v \in C_{0}^{\infty}\left(\mathbb{R}^{n}\right) .
$$

Note that $X_{j}^{*}$ does not have to be a vector field. In general, it is an operator of the form $X_{j}^{*}=X_{j}+f_{j}$, where $f_{j}$ is a suitable smooth function.

If $E=\left(E_{1}, \ldots, E_{k}\right)$ is a vector field, then we simply write $X^{*} \cdot E=\sum_{j=1}^{k} X_{j}^{*} E_{j}$, and call this expression a generalized divergence.

Example (Heisenberg group). In $\mathbb{R}^{3} \equiv \mathbb{C} \times \mathbb{R}$, with points denoted by $\left(x_{1}, x_{2}, t\right)$, or equivalently $(z, t)$, where $z=x_{1}+i x_{2}$, define the group law

$$
\left(z_{1}, t_{1}\right)\left(z_{2}, t_{2}\right)=\left(z_{1}+z_{2}, t_{1}+t_{2}+2 \operatorname{Im}\left(z_{1} \overline{z_{2}}\right)\right) .
$$

Then, $\mathbb{R}^{3}$ becomes a Lie group, usually denoted by $\mathbb{H}_{1}$ (the so-called Heisenberg group). A basis of left invariant vector fields is given by

$$
X_{1}=\frac{\partial}{\partial x_{1}}+2 x_{2} \frac{\partial}{\partial t}, \quad X_{2}=\frac{\partial}{\partial x_{2}}-2 x_{1} \frac{\partial}{\partial t}, \quad T=\frac{\partial}{\partial t} .
$$

Since $X_{1}, X_{2}$, and $\left[X_{1}, X_{2}\right]=-4 \frac{\partial}{\partial t}$ span the tangent space $\mathbb{R}^{3}$ at every point, we see that the system consisting of two vector fields $X_{1}, X_{2}$ satisfies Hörmander's condition. An important property of the Heisenberg group is that Lebesgue measure coincides with the left and right invariant Haar measure. Heisenberg group the simplest nontrivial example of the so-called Carnot groups (known also as stratified groups). For a deeper treatment of this topic see e.g. Folland and Stein [22], and Stein [66].

With a family of vector fields satisfying Hörmander's condition one can associate a suitable metric that we next describe. We say that an absolutely continuous curve $\gamma:[a, b] \rightarrow \mathbb{R}^{n}$ is admissible if there exist functions $c_{j}(t)$, $a \leq t \leq b$ satisfying

$$
\sum_{j=1}^{k} c_{j}(t)^{2} \leq 1 \quad \text { and } \quad \dot{\gamma}(t)=\sum_{j=1}^{k} c_{j}(t) X_{j}(\gamma(t)) .
$$

Note that if the vector fields $X_{j}$ are not linearly independent at some point, then the coefficients $c_{j}$ are not unique.

The distance $\rho(x, y)$ is defined as the infimum of those $T>0$ for which there exists an admissible curve $\gamma:[0, T] \rightarrow \mathbb{R}^{n}$ with $\gamma(0)=x$ and $\gamma(T)=y$. This metric is known in the literature under many different names. We like the name Carnot-Carathéodory metric and use it throughout this paper. A priori it is not clear whether this is a metric i.e. it is not clear whether one can connect any two points of $\mathbb{R}^{n}$ by an admissible curve. This is however the contents of the theorem of Chow, [10]. For more recent proofs, see e.g. Gromov, [36], Nagel, Stein and Wainger [61] or Varopoulos, Saloff-Coste and Coulhon [73]. Balls with respect to metric $\rho$ are called metric balls and denoted by $\widetilde{B}$. Moreover, $\operatorname{diam} F$ will always denote the diameter of a set $F$ with respect to the metric $\rho$.

Nagel, Stein and Wainger [61] proved many important properties of this metric that we next recall (for proofs see also [36], [73, Sect. IV.5]). 
Theorem 2.1. Let $X_{1}, \ldots, X_{k}$ be as above. Then for every relatively compact open set $\Omega \subset \subset \mathbb{R}^{n}$ there are constants $C_{1}, C_{2}$ and $\lambda \in(0,1]$ such that

$$
C_{1}|x-y| \leq \rho(x, y) \leq C_{2}|x-y|^{\lambda}
$$

for every $x, y \in \Omega$.

Thus, identity map gives a homeomorphism between $\left(\mathbb{R}^{n}, \rho\right)$ and $\mathbb{R}^{n}$ with the Euclidean metric.

Moreover if $\Omega$ is bounded with respect to the Euclidean metric, then it is bounded with respect to $\rho$. Unfortunately the converse to the last statement is, in general, false. Indeed, if one of the vector fields is $x_{1}^{2} \partial / \partial x_{1}$, then it is easy to see that the Carnot-Carathéodory distance to infinity is finite. The problem is caused by the rapid growth of the coefficient of the vector field.

This leads to some technical problems (which are not very difficult to cope with, and appear mostly on the notational level). However, we would like to avoid them. The following result of Garofalo and Nhieu, [33, Proposition 2.11] shows when such an unpleasant phenomenon does not occur.

Proposition 2.2. Let $X_{1}, X_{2}, \ldots, X_{k}$ be as above and suppose that in addition, they have coefficients which are globally Lipschitz on $\mathbb{R}^{n}$. Then a subset of $\mathbb{R}^{n}$ is bounded with respect to the Carnot-Carathéodory metric if and only if it is bounded with respect to the Euclidean metric.

The same claim holds for Carnot groups, see e.g. [33, Proposition 2.8].

To avoid the technical problems mentioned above we assume in the remaining part of the paper that the above holds i.e., we assume that a subset of $\mathbb{R}^{n}$ is bounded with respect to the Carnot-Carathéodory metric if and only if it is bounded with respect to the Euclidean metric.

Now we can state the second part of the result of Nagel, Stein and Wainger.

Theorem 2.3. Let $X_{1}, X_{2}, \ldots, X_{k}$ be as above. Then for every bounded open set $\Omega \subset \mathbb{R}^{n}$ there exists $C \geq 1$ such that one has

$$
|\widetilde{B}(x, 2 r)| \leq C|\widetilde{B}(x, r)|
$$

whenever $x \in \Omega$ and $r \leq 5 \operatorname{diam} \Omega$.

Without the above additional assumption, inequality (6) holds only for $\Omega$ bounded with respect to the Euclidean metric and $r<r_{0}$ for some $r_{0}$ depending on $\Omega$. We could have $r_{0}<\operatorname{diam} \Omega$, since it could happen that $\widetilde{B}(x, \operatorname{diam} \Omega)$ "touches infinity".

Sanchez-Calle [64], and independently Nagel, Stein and Wainger [61], proved that in any bounded domain $\Omega \subset \mathbb{R}^{n}$ there exists a fundamental solution of the sub-Laplacian

$$
\Delta_{X}=-\sum_{j=1}^{k} X_{j}^{*} X_{j},
$$

with growth properties generalizing the well known ones for the fundamental solution of the classical Laplace operator. 
By the fundamental solution we mean a function $\Gamma(x, y)$ defined in $\Omega \times \Omega$, smooth off the diagonal and such that for every $\varphi \in C_{0}^{\infty}(\Omega)$ we have

$$
\left(\Delta_{X} \int_{\Omega} \Gamma(\cdot, y) \varphi(y) d y\right)(x)=\varphi(x) .
$$

The growth estimates of [64] and [61] imply that, for $n \geq 3$, we have

$$
C^{-1} \frac{\rho(x, y)^{2}}{|\widetilde{B}(x, \rho(x, y))|} \leq \Gamma(x, y) \leq C \frac{\rho(x, y)^{2}}{|\widetilde{B}(x, \rho(x, y))|},
$$

and, for $n \geq 2$,

$$
\left|X_{i} \Gamma(x, y)\right| \leq C \frac{\rho(x, y)}{|\widetilde{B}(x, \rho(x, y))|}, \quad\left|X_{i} X_{j} \Gamma(x, y)\right| \leq \frac{C}{|\widetilde{B}(x, \rho(x, y))|}
$$

for all $x, y \in \Omega$. In the last two inequalities each of the differentiations can be performed either with respect to $x$ or to $y$.

In the proof of the main result (Theorem 3.1) we apply only the estimates for the derivatives of $\Gamma$, so the result holds for all Euclidean dimensions $n \geq 2$.

Example (continued). Abusing slightly the notation, we shall abbreviate $x=$ $(z, t)$. Define a continuous homogeneous norm on the Heisenberg group $\mathbb{H}_{1}$ by $\|x\|=\rho(0, x)$, where $\rho$ is the Carnot-Carathéodory metric associated with vector fields $X_{1}, X_{2}$. For $r>0$ define a dilation $\delta_{r}$ by $\delta_{r}(z, t)=\left(r z, r^{2} t\right)$. Dilations form a group of automorphisms of $\mathbb{H}_{1}$. The homogeneous norm has the following properties:

(i) $\quad\|\cdot\|: \mathbb{H}_{1} \rightarrow \mathbb{R}_{+}$is continuous;

(ii) $\left\|x^{-1}\right\|=\|x\|$;

(iii) $\left\|\delta_{r} x\right\|=r\|x\|$ for all $r>0$;

(iv) $\|x\|=0$ if and only if $x=0$.

Since the metric $\rho$ is left invariant, it can be recovered from $\|\cdot\|$ by $\rho(x, y)=$ $\left\|x^{-1} y\right\|$. Note also that $\rho$ commutes with the dilations, i.e. $\rho\left(\delta_{r} x, \delta_{r} y\right)=r \rho(x, y)$. Now it is an exercise to prove Theorems 2.1 and 2.3 in this particular situation. Namely there exists a constant $C_{2}>0$ and for every bounded open set $\Omega \subset \mathbb{H}_{1}$ there exists a constant $C_{1} \geq 1$ such that

$$
\begin{gathered}
C_{1}^{-1}|x-y| \leq \rho(x, y) \leq C_{1}|x-y|^{1 / 2} \quad \text { whenever } x, y \in \Omega, \\
|\widetilde{B}(x, r)|=C_{2} r^{4} \quad \text { for every } x \in \mathbb{H}_{1} \text { and } r>0 .
\end{gathered}
$$

One can also prove that the Hausdorff dimension of $\mathbb{H}_{1}$ with respect to the metric $\rho$ is equal to 4, see Mitchell [57].

In order to get a glimpse of the behaviour of $\rho$, consider another homogeneous norm $\|\cdot\|^{\prime}$, given by the formula $\|(z, t)\|^{\prime}=\left(t^{2}+|z|^{4}\right)^{1 / 4}$. Obviously, it satisfies the conditions (i)-(iv). Moreover, $d(x, y):=\left\|x^{-1} y\right\|^{\prime}$ is a metric which commutes with dilations. The equivalence of $d$ and $\rho$ is easy to prove; it follows from the left invariance of both metrics and the fact that they commute with dilations. 
Note that no Riemannian metric is equivalent to the Carnot-Carathéodory metric on $\mathbb{H}_{1}$.

As was shown by Folland [20] (see also [21], [49]), the fundamental solution for the sub-Laplacian

$$
X_{1}^{2}+X_{2}^{2}=\left(\frac{\partial}{\partial x_{1}}+2 x_{2} \frac{\partial}{\partial t}\right)^{2}+\left(\frac{\partial}{\partial x_{2}}-2 x_{1} \frac{\partial}{\partial t}\right)^{2}
$$

is given by $\Gamma\left(\left(z_{1}, t_{1}\right),\left(z_{2}, t_{2}\right)\right)=G\left(\left(z_{1}, t_{1}\right)\left(z_{2}, t_{2}\right)^{-1}\right)$, where

$$
G(z, t)=\frac{\text { const }}{\left(t^{2}+|z|^{4}\right)^{1 / 2}} .
$$

Multiplying both sides of (7) by $u(x) \in C_{0}^{\infty}(\Omega)$, integrating with respect to $x$ and next integrating by parts we easily obtain the following well known representation formula.

Lemma 2.4. Let $\Omega \subset \mathbb{R}^{n}$ be a bounded domain. Then for every $u \in C_{0}^{\infty}(\Omega)$, and for every $x \in \Omega$ one has

$$
u(x)=\int_{\Omega} X_{y} \Gamma(y, x) \cdot X_{y} u(y) d y .
$$

By a cut-off function we mean a function $\varphi$ which is identically equal to 1 on some metric ball, vanishes outside the twice enlarged metric ball, and such that the length of its gradient $|X \varphi|$ is bounded by a constant times the inverse of the radius of this ball. Using the distance function, it is easy to construct such a function with a bound for the Lipschitz constant instead of the pointwise bound for the gradient. However, it is not a priori obvious that the distance function is differentiable in any sense. Indeed, due to Theorem 2.1, the distance function is only Hölder continuous. The fundamental solution $\Gamma$ has decay properties similar to those of $\rho$. Citti, Garofalo and Lanconelli, [13], used this property to construct smooth cut-off functions.

Lemma 2.5. Let $X_{1}, \ldots, X_{k}$ be as above. Then, given open and bounded set $\Omega \subset$ $\mathbb{R}^{n}$, there exists a constant $C \geq 0$ such that for every $x \in \Omega$ and $t \leq \operatorname{diam} \Omega$, one can find a function $\varphi \in C_{0}^{\infty}(\widetilde{B}(x, t))$ satisfying $0 \leq \varphi \leq 1, \varphi=1$ on $\widetilde{B}(x, t / 2)$ and $|X \varphi| \leq C t^{-1}$.

Recently Franchi, Serapioni, and Serra Cassano [29] and Garofalo and Nhieu [33] have independently proved weak differentiability of Lipschitz functions along given vector fields, so one can construct cut-off functions starting directly from the distance function.

With a family of Hörmander vector fields we can associate a Sobolev space on an open set $\Omega \subset \mathbb{R}^{n}$ as follows

$$
W_{X}^{1, p}(\Omega)=\left\{u \in L^{p}(\Omega): X_{j} u \in L^{p}(\Omega) \text { for } j=1,2, \ldots, k\right\} .
$$


The derivatives are understood in the distributional sense and the space is equipped with the norm $\|u\|_{1, p}=\|u\|_{p}+\|X u\|_{p}$. It was proved independently by Franchi, Serapioni, and Serra Cassano [29] and by Garofalo and Nhieu [34] that $C^{\infty}$ functions are dense in $W_{X}^{1, p}(\Omega)$. However, the main idea of the proof goes back to an old paper of Friedrichs [30]. Besides, Sobolev functions with compact support in $\Omega$ can be approximated by compactly supported smooth functions.

Given a bounded open set $\Omega \subset \mathbb{R}^{n}$ we say that $Q$ is a homogeneous dimension relative to $\Omega$ if for some positive constant $C$ we have

$$
\frac{|\widetilde{B}|}{\left|\widetilde{B}_{0}\right|} \geq C\left(\frac{r}{r_{0}}\right)^{Q}
$$

where $\widetilde{B}_{0}$ is an arbitrary ball centered at some point of $\Omega$ of radius $r_{0} \leq \operatorname{diam} \Omega$, and $\widetilde{B}=\widetilde{B}(x, r), x \in \widetilde{B}_{0}, r \leq r_{0}$.

It is well known that doubling property implies the existence of such a $Q$, see Lemma 2.7 below. However, $Q$ is not unique and it may change with $\Omega$. Note that any $Q^{\prime} \geq Q$ is also a homogeneous dimension. In the Heisenberg group example we clearly have the smallest such $Q=4$, and hence $G(z, t)=$ const $\cdot\left(\|(z, t)\|^{\prime}\right)^{2-Q}$, which looks more familiar than (8).

There are plenty of generalizations of the Sobolev imbedding theorem to the setting of vector fields. The statement of Theorem 2.6 below can be found for example in Franchi, Lu, and Wheeden [27], or in Capogna, Danielli, and Garofalo [8]. Various versions of this theorem appear also in Biroli and Mosco [3], Franchi [23], Franchi and Lanconelli [26], Franchi, Lu, and Wheeden [28], Lu [50], [51] Garofalo and Nhieu [34], Hajlasz and Koskela [38], [39], Jerison [43], Maheux and Saloff-Coste [56], Saloff-Coste [63], Varopoulos [72], Varopoulos, Saloff-Coste, and Coulhon [73].

Theorem 2.6. Let $X_{1}, \ldots, X_{k}$ be as before. Let $Q$ be a homogeneous dimension relative to $\Omega$. Given $1 \leq p<Q$ there is a constant $C>0$ such that for every $\widetilde{B}=\widetilde{B}(x, r), x \in \Omega$, and $r \leq \operatorname{diam} \Omega$ we have

$$
\left(f_{\widetilde{B}}\left|u-u_{\widetilde{B}}\right|^{p^{*}} d x\right)^{1 / p^{*}} \leq C r\left(f_{\widetilde{B}}|X u|^{p} d x\right)^{1 / p},
$$

where $p^{*}=Q p /(Q-p)$.

The imbedding for compactly supported functions is easier and has been obtained in Rotschild and Stein [62], Capogna, Danielli, and Garofalo [5], [6], Danielli [16], and Franchi, Gallot, and Wheeden [24].

Note that Sobolev inequality (11) implies the Poincaré inequality

$$
\left(f_{\widetilde{B}}\left|u-u_{\widetilde{B}}\right|^{p} d x\right)^{1 / p} \leq C r\left(f_{\widetilde{B}}|X u|^{p} d x\right)^{1 / p},
$$

for any $1 \leq p<\infty$. Indeed, if $1 \leq p<Q$, then it suffices to apply Hölder inequality. If $1 \leq p<\infty$ is arbitrary then, as we have already noticed, any 
number $Q^{\prime}>Q$ is a homogeneous dimension relative to $\Omega$, so we always can find $Q^{\prime}>p$ and proceed as above.

Remark. It is much more surprising that one can also deduce Sobolev imbedding (11) from the Poincare inequality (12). It seems that for some particular situations this fact was first observed independently by Grigor'yan [35], and by Saloff-Coste [63]. Later, the same has also been established in a very general setting by many other authors, Biroli and Mosco [3], Franchi, Lu and Wheeden [28], Garofalo and Nhieu [34], Hajkasz and Koskela [38], [39], Maheux and Saloff-Coste [56], and Sturm [69].

\subsection{Whitney decomposition and partition of unity}

Let $Y$ be a metric space with metric $\rho$ and some positive Borel measure $\mu$. Assume that $\mu$ is finite on bounded sets. We say that $\mu$ is doubling on $\Omega \subset Y$ if there is a constant $C_{d} \geq 1$ such that

$$
\mu(B(x, 2 r)) \leq C_{d} \mu(B(x, r)),
$$

whenever $x \in \Omega$ and $r<5 \operatorname{diam} \Omega$. Note that, under the assumptions of Theorem 2.3, the Lebesgue measure is doubling on any bounded open subset of $\mathbb{R}^{n}$ with respect to the Carnot-Carathéodory metric.

In this paper we are concerned with vector fields satisfying Hörmander's condition. Nevertheless, we prefer to state auxiliary technical results - whenever it is possible - for the general case of a metric space $Y$. This shows much better which results depend on the doubling property only, and which on the other hand require more sophisticated information about vector fields.

It is well known that doubling condition implies the lower bound for the growth of the measure of the ball.

Lemma 2.7. Let $\mu$ be a Borel measure on a metric space $Y$, finite on bounded sets. Assume that $\mu$ is doubling on a bounded open set $\Omega \subset Y$. Then

$$
\mu(B(x, r)) \geq(2 \operatorname{diam} \Omega)^{-s} \mu(\Omega) r^{s},
$$

for $s=\log _{2} C_{d}, x \in \Omega$ and $r \leq \operatorname{diam} \Omega$.

This applies to Hörmander vector fields and implies (10) with $Q$ computed from the doubling constant.

The familiar Whitney decomposition of an open set in $\mathbb{R}^{n}$ and an associated partition of unity has been generalized to the setting of a metric space equipped with a doubling measure by Coifman and Weiss [15], and Macías and Segovia [55], respectively. By now it is a standard technique. For the sake of completness we recall it with some details.

Let, as before $\Omega \subset Y, \Omega \neq Y$ be open; assume $\mu$ doubling on $\Omega$. For $x \in \Omega$ define $r_{x}=\operatorname{dist}\left(x, \Omega^{c}\right) / 1000$. Then $\left\{B\left(x, r_{x}\right)\right\}_{x \in \Omega}$ is a covering of $\Omega$. Select a maximal subfamily of pairwise disjoint balls and denote it by $\left\{B\left(x_{i}, r_{i}\right)\right\}_{i \in I}$. It 
follows from the maximality that $\bigcup_{i \in I} B\left(x_{i}, 3 r_{i}\right)=\Omega$. Moreover the doubling property implies that there exists an integer $N \geq 1$ (depending only on the doubling constant $C_{d}$ ) such that no point of $\Omega$ belongs to more than $N$ balls $B\left(x_{i}, 6 r_{i}\right)$.

Now with such a (Whitney) decomposition of $\Omega$ into balls one can associate a Lipschitz partition of unity that we next describe. Let $\psi$ be a real smooth function with $\psi \equiv 1$ on $[0,1], \psi \equiv 0$ on $\left[\frac{4}{3}, \infty\right), 0 \leq \psi \leq 1$. Put

$$
\varphi_{i}(x)=\psi\left(\frac{\operatorname{dist}\left(x, x_{i}\right)}{3 r_{i}}\right) .
$$

Then $\varphi_{i} \equiv 1$ on $B\left(x_{i}, 3 r_{i}\right), \varphi_{i} \equiv 0$ off $B\left(x_{i}, 4 r_{i}\right)$ and the Lipschitz constant of $\varphi_{i}$ is $C r_{i}^{-1}$. We define a partition of unity in a standard way,

$$
\theta_{i}(x):=\frac{\varphi_{i}(x)}{\sum_{k} \varphi_{k}(x)} .
$$

Note that the sum in the denominator is locally finite - in fact, for any point $x$ at most $N$ terms are nonzero.

Obviously $\sum_{i} \theta_{i}(x) \equiv 1$ on $\Omega$, and $\operatorname{supp} \theta_{i} \subset B\left(x_{i}, 6 r_{i}\right)$. Moreover $\theta_{i}$ is Lipschitz with the Lipschitz constant $c / r_{i}$, where $c$ depends on the doubling constant $C_{d}$ only. (The proof of Lipschitz estimate for $\theta_{i}$ is easy; one has to remember that (a) the denominator in (13) is not less than 1 , and (b) if both $\varphi_{i}$ and $\varphi_{k}$ do not vanish at some point then their Lipschitz constants are comparable.)

In the case when the metric space $Y$ coincides with $\mathbb{R}^{n}$ (equipped with the Carnot-Carathéodory metric), an open set is bounded and $\mu$ is the Lebesgue measure, we can additionally assume with no loss of generality that all $\theta_{i}$ are smooth and $\left|X \theta_{i}\right| \leq C r_{i}^{-1}$. This follows from the construction of the partition of unity and existence of smooth cut-off functions, Lemma 2.5 . We will apply this construction in the proof of Lemma 3.2.

\subsection{Fractional Integration Theorem}

In this section we state a version of the Fractional Integration Theorem obtained by Hajłasz and Koskela [39].

Assume that $Y$ is a metric space and let $\mu$ be a Borel measure on $Y$ which is strictly positive on every ball. For $\Omega \subset Y$ bounded and open, $p>0, \sigma \geq 1$, and $\alpha>0$ we define

$$
J_{\alpha, p}^{\sigma, \Omega} g(x)=\sum_{2^{i} \leq 2 \sigma \operatorname{diam} \Omega} 2^{i \alpha}\left(f_{B\left(x, 2^{i}\right)}|g|^{p} d \mu\right)^{1 / p} .
$$

The counterpart of fractional integration theorem for these abstract 'Riesz potential' operators reads as follows. 
Theorem 2.8. Let $\Omega \subset Y$ be an open and bounded set and $1 \leq \sigma<\infty$. Assume that the measure $\mu$ is doubling on $V=\{x \in Y: \operatorname{dist}(x, \Omega)<2 \sigma \operatorname{diam} \Omega\}$. Moreover assume that for some constants $b, s>0$ we have

$$
\mu(B(x, r)) \geq b\left(\frac{r}{\operatorname{diam} \Omega}\right)^{s} \mu(\Omega)
$$

whenever $x \in \Omega$ and $r \leq 2 \sigma \operatorname{diam} \Omega$.

If $\alpha>0$, and $0<p<q<s / \alpha$, then

$$
\left\|J_{\alpha, p}^{\sigma, \Omega} g\right\|_{L^{q^{*}}(\Omega, \mu)} \leq C(\operatorname{diam} \Omega)^{\alpha} \mu(\Omega)^{-\alpha / s}\|g\|_{L^{q}(V, \mu)},
$$

where $q^{*}=s q /(s-\alpha q)$ and $C=C\left(\alpha, \sigma, p, q, b, s, C_{d}\right)$.

\section{Main result}

As we have already said in the introduction, we are concerned with the regularity of subelliptic $p$-harmonic maps into spheres. These are the critical points (with respect to variations in the range) of the subelliptic $p$-energy functional defined by (3).

To be more precise, assume that $X_{1}, \ldots X_{k}$ are smooth vector fields satisfying Hörmander's condition in $\mathbb{R}^{n}$. From now on, $\Omega$ shall denote a bounded domain in $\mathbb{R}^{n}$. Assume also that a map $u=\left(u_{1}, u_{2}, \ldots, u_{m+1}\right)$ from $\Omega$ to $S^{m}$, the unit Euclidean sphere in $\mathbb{R}^{m+1}$, belongs to $W_{X}^{1, p}\left(\Omega, S^{m}\right)$, i.e. all distributional derivatives $X_{i} u_{j}$ belong to $L^{p}(\Omega)$ and moreover $\sum_{i}\left(u_{i}\right)^{2} \equiv 1$ a.e. Then, $u$ is said to be a subelliptic p-harmonic map if and only if for every compactly supported smooth test map $\psi=\left(\psi_{1}, \psi_{2}, \ldots, \psi_{m+1}\right)$ one has

$$
\left.\frac{d}{d t}\right|_{t=0} E_{p}\left(u_{t}\right)=0
$$

where $u_{t}=\frac{u+t \psi}{|u+t \psi|}$ is the nearest point projection of $u+t \psi$ onto $S^{m}$. An elementary computation of the derivative leads to

$$
\begin{aligned}
\left.\frac{d}{d t}\right|_{t=0} E_{p}\left(u_{t}\right) & =p \int_{\Omega}|X u|^{p-2}\left\langle X u ;\left.X \frac{d}{d t}\right|_{t=0} u_{t}\right\rangle d x \\
& =p \int_{\Omega}|X u|^{p-2}\langle X u ; X(\psi-(u \cdot \psi) u)\rangle d x \\
& =p \int_{\Omega}|X u|^{p-2}\langle X u ; X \psi\rangle d x-p \int_{\Omega}|X u|^{p} u \cdot \psi d x
\end{aligned}
$$

The last equality follows from the constraint $|u| \equiv 1$, which implies that $u$ is orthogonal to $X_{j} u$ for any choice of $j$.

Thus, subelliptic $p$-harmonic maps coincide with those (weak) solutions of the nonlinear system of equations

$$
-X^{*} \cdot\left(|X u|^{p-2} X u\right)=|X u|^{p} u,
$$


which satisfy the constraint $\sum_{i}\left(u_{i}\right)^{2} \equiv 1$. In the Euclidean case $k=n, X_{j}=$ $\partial / \partial x_{j}$, this system has the familiar form $-\operatorname{div}\left(|\nabla u|^{p-2} \nabla u_{i}\right)=|\nabla u|^{p} u_{i}$, where $i=1,2, \ldots, m+1$. Note that for weak solutions an appropriate integral identity,

$$
\int_{\Omega} \sum_{j=1}^{k}|X u|^{p-2} X_{j} u X_{j} \psi=\int_{\Omega}|X u|^{p} u \psi
$$

holds not only for every $\psi \in C_{0}^{\infty}\left(\Omega, \mathbb{R}^{m+1}\right)$, but also for every $\psi \in W_{X}^{1, p}(\Omega)$ with compact support in $\Omega$. This follows from the density results mentioned in Sect. 2.1.

Denote by $Q$ the homogeneous dimension (relative to $\Omega$ ) associated with the $X_{j}$ 's. From now on we assume that $p=Q$. Our main result reads as follows.

Theorem 3.1. Every subelliptic $Q$-harmonic map $u \in W_{X}^{1, Q}\left(\Omega, S^{m}\right)$ is locally Hölder continuous.

Both the theorem and its proof have local nature and thus, the same result holds if the Hörmander vector fields are defined on $\Omega$ only. We impose some global assumptions on the vector fields on $\mathbb{R}^{n}$ only to simplify notation.

The proof of this theorem consists of three steps. (1) First, we exploit the constraint $|u| \equiv 1$ to rewrite the Euler-Lagrange system (16) in an equivalent form. It turns out that the right side resembles the familiar expression $E \cdot B$, where one of the two vector fields is divergence free, and the other one is a gradient field. (2) Next, the ghost of Hardy space enters: we show in Lemma 3.2 that it is possible to integrate the right side against $\eta(u-$ const), where $\eta$ is a smooth cutoff function (which behaves nicely on metric balls). This is the crucial part of the proof and the main novelty of this paper. Then, after a rather standard computation which involves a hole filling argument, we arrive at a Caccioppoli type estimate: it turns out that - as $r$ goes to zero $-\int_{B(x, r)}|X u|^{Q}$ goes to zero faster than some fixed, positive power of $r$. (3) In the final step we apply an abstract, metric version of the classical Dirichlet Growth Theorem and obtain Hölder continuity of $u$.

Proof. To begin with, note that a well-known trick, used by Frédéric Hélein to rewrite the harmonic map equation in dimension 2, still works. Set $V_{i}=$ $|X u|^{Q-2} X u_{i}$. The constraint condition $\sum_{l} u_{l}^{2}=1$ implies $\sum_{l} u_{l} V_{l}=0$, hence

$$
V_{i}=\sum_{l=1}^{m+1} u_{l}\left(u_{l} V_{i}-u_{i} V_{l}\right)
$$

By a straightforward computation one checks that $X^{*} \cdot\left(u_{l} V_{i}-u_{i} V_{l}\right)=0$. Indeed, it suffices to show that $X^{*} \cdot\left(u_{l} V_{i}\right)$ can be written as an expression symmetric with respect to $i$ and $l$. Multiplying by $\psi \in C_{0}^{\infty}$, integrating and invoking (16), we obtain 


$$
\begin{aligned}
\int_{\Omega} u_{l} V_{i} \cdot X \psi & =\int_{\Omega} V_{i} \cdot X\left(u_{l} \psi\right)-\int_{\Omega} \psi V_{i} \cdot X u_{l} \\
& =\int_{\Omega}|X u|^{Q} u_{i} u_{l} \psi-\int_{\Omega} \psi|X u|^{Q-2} X u_{i} \cdot X u_{l} .
\end{aligned}
$$

The right hand side of this identity is obviously symmetric with respect to $i$ and $l$, and the claim follows. Taking now the generalized divergence of both sides of (18), we obtain

$$
X^{*} \cdot\left(|X u|^{Q-2} X u_{i}\right)=\sum_{l=1}^{m+1} X^{*} \cdot\left(u_{l} E_{i, l}\right),
$$

where $E_{i, l} \equiv\left(u_{l} V_{i}-u_{i} V_{l}\right) \in L^{Q /(Q-1)}$ is of zero generalized divergence, $X^{*}$. $E_{i, l}=0$. To derive a Caccioppoli type estimate, we integrate (19) against a suitable test function constructed from $u$.

In a more classical situation as in Hélein [40], or Strzelecki [67], the test function $\eta$ (u-const) belongs to $W^{1, Q}\left(B^{Q}\right) \subset \mathrm{BMO}$, and the right hand side of (19) belongs locally to $\mathscr{H}^{1}$ due to the theorem of Coifman, Lions, Meyer and Semmes, [14]. Thus one can integrate (19) against such a test function, employing the duality of $\mathscr{H} \mathscr{C}^{1}$ and BMO.

Here the theory of Hardy spaces is not available but, as we shall see, a suitable duality inequality still holds. In the proof of the duality inequality stated below we follow some ideas of Coifman, Lions, Meyer and Semmes [14], Hajrasz and Koskela [39], and Semmes [65].

Lemma 3.2 (duality inequality). For any pair of indices $i, l$, and any metric ball $\widetilde{B} \subset 200 \widetilde{B} \subset \Omega$, the inequality

$$
\left|\int_{\widetilde{B}} X^{*} \cdot\left(u_{l} E_{i, l}\right)(x) \varphi(x) d x\right| \leq C\|X u\|_{L^{Q}(100 \widetilde{B})}^{Q}\|X \varphi\|_{L^{Q}(\widetilde{B})}
$$

holds for every $\varphi \in W_{X}^{1, Q}(\widetilde{B})$ with compact support. The constant $C$ does not depend on $\widetilde{B}$.

Proof. It is enough to prove the lemma for smooth compactly supported $\varphi$. In the sequel, we drop the subscripts $i$ and $l$. Fix a smooth cut-off function $\eta$ with $\eta \equiv 1$ on $2 \widetilde{B}, \eta \equiv 0$ off $4 \widetilde{B},|X \eta| \leq C(\operatorname{diam} \widetilde{B})^{-1}$. By the representation formula, see Lemma 2.4, we obtain

$$
\begin{aligned}
\int_{\widetilde{B}} X_{x}^{*} \cdot(u E)(x) \varphi(x) d x & =\int X_{x}^{*} \cdot(u E)(x) \eta(x) \varphi(x) d x \\
& =\iint X_{x}^{*} \cdot(u E)(x) \eta(x) X_{y} \Gamma(y, x) X_{y} \varphi(y) d y d x
\end{aligned}
$$

Here, the subscripts $x$ and $y$ denote the variable with respect to which the differentiation is performed. Set

$$
A(y)=\int X_{x}^{*} \cdot(u E)(x) \eta(x) X_{y} \Gamma(y, x) d x .
$$


We claim that

$$
\|A\|_{L^{Q /(Q-1)(\widetilde{B})}} \leq C\|X u\|_{L^{Q(100 \widetilde{B})}}^{Q} .
$$

Of course, the lemma follows from (20) and Hölder inequality. The idea of the proof of inequality (20) is to estimate $A$ by a generalized Riesz potential of $|X u|^{Q} \in L^{1}$, say $|A| \leq C J_{1, p}\left(|X u|^{Q}\right)$ for some $p<1$, and then to apply a generalized fractional integration theorem (Theorem 2.8) which implies that $J_{1, p}^{\cdots}: L^{1} \rightarrow L^{Q /(Q-1)}$.

Note that the above version of the fractional integration theorem provides a replacement for the theory of Hardy spaces. Indeed, in general, the classical Riesz potential $I_{1}$ of a $L^{1}$ function on $\mathbb{R}^{n}$ does not belong to $L^{n /(n-1)}$ unless the function belongs to the Hardy space. As we obtain estimates by a better Riesz potential, we do not need Hardy spaces any more.

Now it remains to prove the desired estimate for $A$. Here are the details.

Fix $y \in \widetilde{B}$ and let $\left\{\theta_{i}^{y}\right\}_{i \in I}$ be a smooth partition of unity associated to the Whitney decomposition of $\Omega \backslash\{y\}$ (with respect to the Carnot-Carathéodory metric, see Sect. 2.2). Write $\widetilde{B}_{i}=\widetilde{B}\left(x_{i}, 6 r_{i}\right)$ to denote the Whitney ball which supports $\theta_{i}^{y}$. (In the sequel, we drop the superscript $y$.) Recalling that $X^{*} \cdot E=0$, and integrating by parts, we obtain

$$
\begin{aligned}
A(y) & =\sum_{i \in I} \int_{\widetilde{B}_{i}} X_{x}^{*} \cdot(u E)(x) \eta(x) \theta_{i}(x) X_{y} \Gamma(y, x) d x \\
& =\sum_{i \in I} \int_{\widetilde{B}_{i}} X_{x}^{*} \cdot\left(\left(u-u_{\widetilde{B}_{i}}\right) E\right)(x) \eta(x) \theta_{i}(x) X_{y} \Gamma(y, x) d x \\
& =-\sum_{i \in I} \int_{\widetilde{B}_{i}}\left(u(x)-u_{\widetilde{B}_{i}}\right) E(x) \cdot X_{x}\left[\eta(x) \theta_{i}(x) X_{y} \Gamma(y, x)\right] d x .
\end{aligned}
$$

Pick $I^{\prime} \subset I$ which consists of all the indices such that $\operatorname{supp} \theta_{i} \cap 4 \widetilde{B} \neq \emptyset$. Note that $|X \eta| \neq 0$ in the annulus $4 \widetilde{B} \backslash 2 \widetilde{B}$ only. Hence, $|X \eta(x)| \leq C \rho(x, y)^{-1}$ for every $x$. We also have $\left|X \theta_{i}(x)\right| \leq C \rho(x, y)^{-1}$ for $i \in I^{\prime}$. Therefore, the bounds for the derivatives of the fundamental solution $\Gamma(y, x)$, see Sect. 2.1 , directly imply that for $i \in I^{\prime}$

$$
\left|X_{x}\left[\eta(x) \theta_{i}(x) X_{y} \Gamma(y, x)\right]\right| \leq C|\widetilde{B}(y, \rho(x, y))|^{-1} .
$$

Now, the doubling condition implies that, for $x \in \widetilde{B}_{i}$ and $i \in I^{\prime}$, the right hand side of (22) is comparable to $\left|\widetilde{B}_{i}\right|^{-1}$. Since the terms in the sum (21) are equal to zero for $i \notin I^{\prime}$ we obtain

$$
\begin{aligned}
|A(y)| & \leq C \sum_{i \in I^{\prime}} f_{\widetilde{B}_{i}}\left|u-u_{\widetilde{B}_{i}}\right||E| d x \\
& \leq C \sum_{i \in I^{\prime}}\left(f_{\widetilde{B}_{i}}\left|u-u_{\widetilde{B}_{i}}\right|^{Q^{2}} d x\right)^{1 / Q^{2}}\left(f_{\widetilde{B}_{i}}|E|^{Q^{2} /\left(Q^{2}-1\right)} d x\right)^{\left(Q^{2}-1\right) / Q^{2}} \\
& \leq C \sum_{i \in I^{\prime}} r_{i}\left(f_{\widetilde{B}_{i}}|X u|^{Q^{2} /(Q+1)} d x\right)^{(Q+1) / Q} .
\end{aligned}
$$


In the last inequality, we have applied Sobolev inequality (Theorem 2.6) for $p=\frac{Q^{2}}{Q+1}$ and the estimate $|E| \leq C|X u|^{Q-1}$. Now it is not surprising that the right hand side of the last inequality can be bounded by the generalized Riesz potential $J_{1, p}^{2,8 \widetilde{B}}\left(|X u|^{Q}\right)$, where $p=Q /(Q+1)<1$ (see Sect. 2.3 for definition).

Let $i \in I^{\prime}$. If $x_{i} \in \widetilde{B}\left(y, 2^{k-1}\right) \backslash \widetilde{B}\left(y, 2^{k-2}\right)$, for some $k \in \mathbb{Z}$, then $r_{i} \approx 2^{k}$, $\widetilde{B}_{i} \subset \widetilde{B}\left(y, 2^{k}\right)$, and by the doubling property we have $\left|\widetilde{B}_{i}\right| \approx\left|\widetilde{B}\left(y, 2^{k}\right)\right|$. Hence

$$
r_{i}\left(f_{\widetilde{B}_{i}}|X u|^{Q^{2} /(Q+1)} d x\right)^{(Q+1) / Q} \leq C 2^{k}\left(f_{\widetilde{B}\left(y, 2^{k}\right)}|X u|^{Q^{2} /(Q+1)} d x\right)^{(Q+1) / Q} .
$$

Moreover the number of those indices $i \in I^{\prime}$ for which $x_{i} \in \widetilde{B}\left(y, 2^{k-1}\right) \backslash$ $\widetilde{B}\left(y, 2^{k-2}\right)$ is bounded by a constant depending on the doubling constant only (because the balls $\frac{1}{6} \widetilde{B}_{i}$ are pairwise disjoint and their radii are comparable to the diameter of the annulus $\widetilde{B}\left(y, 2^{k-1}\right) \backslash \widetilde{B}\left(y, 2^{k-2}\right)$.) Finally, note that when $k$ is large, say $2^{k-2} \geq \operatorname{diam} 8 \widetilde{B}$, then there is no $i \in I^{\prime}$ with $x_{i} \in \widetilde{B}\left(y, 2^{k-1}\right) \backslash \widetilde{B}\left(y, 2^{k-2}\right)$. Thus

$$
|A(y)| \leq C \sum_{2^{k} \leq 4 \operatorname{diam} 8 \widetilde{B}} 2^{k}\left(f_{B\left(y, 2^{k}\right)}|X u|^{Q^{2} /(Q+1)} d x\right)^{(Q+1) / Q}=C J_{1, \frac{Q}{Q+1}}^{2,8 \widetilde{B}}\left(|X u|^{Q}\right)(y) .
$$

Now Theorem 2.8 implies that

$$
J_{1, \frac{Q}{Q+1}}^{2, \widetilde{B}}: L^{1}(100 \widetilde{B}) \rightarrow L^{Q /(Q-1)}(8 \widetilde{B}),
$$

which completes the proof of (20) and of the whole lemma.

Proof of Theorem 3.1 continued. Fix $\Omega_{1} \subset \subset \Omega$ and $R_{0}>0$ such that $\widetilde{B}\left(x, 400 R_{0}\right) \subset \Omega$ for all $x \in \Omega_{1}$. Fix a small metric ball $\widetilde{B}=\widetilde{B}(x, r), x \in \Omega_{1}$, $r<R_{0}$ and integrate both sides of equation (19) against the test function given by

$$
\psi_{i}:=\eta\left(u_{i}-\left(u_{i}\right)_{2 \widetilde{B}}\right),
$$

where $\eta \equiv 1$ on $\widetilde{B}, \eta \equiv 0$ off $2 \widetilde{B}$, and $|X \eta| \leq C r^{-1}$, see Lemma 2.5. After a routine calculation, using Lemma 3.2, Poincaré inequality (12), and bearing in mind that $X \eta \equiv 0$ on $\widetilde{B}$, we obtain

$$
\begin{aligned}
\int_{\widetilde{B}}|X u|^{Q} d x \leq & C \sum_{i} \int_{2 \widetilde{B}}|X u|^{Q-1}\left|u_{i}-\left(u_{i}\right)_{2 \widetilde{B}}\right||X \eta| d x \\
& +\sum_{i, l}\left|\int_{\widetilde{B}} X^{*} \cdot\left(u_{i} E_{i, l}\right) \psi_{i} d x\right| \\
\leq & C\left(\int_{\widetilde{2} \backslash \widetilde{B}}|X u|^{Q} d x\right)^{(Q-1) / Q}\left(\int_{\widetilde{2}}|X u|^{Q} d x\right)^{1 / Q} \\
& +C\left(\int_{200 \widetilde{B}}|X u|^{Q} d x\right) \sum_{i}\left(\int_{\widetilde{2}}\left|X \psi_{i}\right|^{Q} d x\right)^{1 / Q} .
\end{aligned}
$$


By Poincaré inequality we also have

$$
\sum_{i}\left(\int_{2 \widetilde{B}}\left|X \psi_{i}\right|^{Q} d x\right)^{1 / Q} \leq C\left(\int_{\widetilde{B}}|X u|^{Q} d x\right)^{1 / Q} .
$$

Hence, setting $I(r)=\int_{\widetilde{B}(r)}|X u|^{Q} d x$, we can rewrite (23) in the form

$$
\begin{aligned}
I(r) & \leq C(I(2 r)-I(r))^{(Q-1) / Q} I(2 r)^{1 / Q}+C I(200 r) I(2 r)^{1 / Q} \\
& \leq C(I(200 r)-I(r))^{(Q-1) / Q} I(200 r)^{1 / Q}+C I(200 r)^{(Q+1) / Q}
\end{aligned}
$$

Now, we claim that this implies the existence of numbers $r_{0}>0$ and $\lambda \in(0,1)$ (which do not depend on the size and center of $\widetilde{B}$ ) such that

$$
I(r) \leq \lambda I(200 r) \quad \text { for all } r \leq r_{0} .
$$

To prove this, we argue by contradiction. Were (24) false, for any $\lambda \in(0,1)$ we would find an arbitrarily small $r$ with $I(r)>\lambda I(200 r)$, and hence

$$
\lambda I(200 r)<I(r) \leq C(1-\lambda)^{(Q-1) / Q} I(200 r)+C I(200 r)^{(Q+1) / Q} .
$$

In particular for any $\lambda \in[1 / 2,1)$ we would find an arbitrarily small $r$ such that

$$
\frac{1}{2}<C(1-\lambda)^{(Q-1) / Q}+C I(200 r)^{1 / Q} .
$$

By the absolute continuity of integral, this is absurd for $\lambda$ close to 1 and small $r$. Hence, (24) follows.

A standard iteration argument implies now that there exists a number $\gamma \in$ $(0,1)$ such that a decay estimate of the form

$$
I(r) \equiv \int_{\widetilde{B}(x, r)}|X u(y)|^{Q} d y \leq C r^{\gamma}
$$

is satisfied for all $x \in \Omega_{1}$ and all $r \leq r_{0}$. Finally, we apply an abstract version of the so-called Dirichlet Growth Theorem due to Macías and Segovia [55] (Lemma 3.3 and Lemma 3.4), to deduce local Hölder continuity of $u$. For the sake of completness we provide a short proof following Hajrasz and Kinnunen [37].

Let $0<\alpha, \beta<\infty$ and $R>0$. Recall that, for a locally integrable function $f$, the fractional maximal function of $f$ is defined by

$$
\mathscr{M}_{\alpha, R} f(x)=\sup _{0<r<R} r^{\alpha} f_{\widetilde{B}(x, r)}|f| d y .
$$

Moreover, the fractional sharp maximal function of $f$ is defined by

$$
f_{\beta, R}^{\#}(x)=\sup _{0<r<R} r^{-\beta} f_{\widetilde{B}(x, r)}\left|f-f_{\widetilde{B}(x, r)}\right| d y .
$$

If $R=\infty$ we simply write $f_{\beta}^{\#}(x)$.

We need the following. 
Lemma 3.3. Suppose that $f: \Omega \rightarrow[-\infty, \infty]$ is locally integrable and let $0<$ $\beta<\infty$. Then there is a constant $c$ (which depends only on $\beta$ and on the doubling constant) such that for every $R \leq R_{0}$

$$
|f(x)-f(y)| \leq c \rho(x, y)^{\beta}\left(f_{\beta, 2 R}^{\#}(x)+f_{\beta, 2 R}^{\#}(y)\right)
$$

for almost every $x, y \in \Omega_{1}$ such that $\rho(x, y)<R$.

Proof. Let $x \in \Omega_{1}, 0<r<R$ and denote $B_{i}=\widetilde{B}\left(x, 2^{-i} r\right), i=0,1, \ldots$ Let $N$ be the the complement of the set of Lebesgue points (defined with respect to the Carnot-Carathéodory balls!) for $f$ in $\Omega_{1}$. Since the Lebesgue measure is doubling with respect to the Carnot-Carathéodory metric, the Lebesgue theorem implies $|N|=0$, see Coifman and Weiss [15]. For every $x \in \Omega_{1} \backslash N$ we have $f_{B_{i}} \rightarrow f(x)$ as $i$ goes to infinity and hence

$$
\begin{aligned}
\left|f(x)-f_{\widetilde{B}(x, r)}\right| & \leq \sum_{i=0}^{\infty}\left|f_{B_{i+1}}-f_{B_{i}}\right| \\
& \leq \sum_{i=0}^{\infty} \frac{\left|B_{i}\right|}{\left|B_{i+1}\right|} f_{B_{i}}\left|f-f_{B_{i}}\right| \\
& \leq C \sum_{i=0}^{\infty}\left(2^{-i} r\right)^{\beta}\left(2^{-i} r\right)^{-\beta} f_{B_{i}}\left|f-f_{B_{i}}\right| \\
& \leq C r^{\beta} f_{\beta, r}^{\#}(x) .
\end{aligned}
$$

Take $y \in \Omega_{1} \backslash N$ such that $\rho(x, y)<R$. Then $y \in \widetilde{B}(x, r)$ for some $r=$ $(1+\varepsilon) \rho(x, y)<R$. Since $\widetilde{B}(x, r) \subset \widetilde{B}(y, 2 r)$ we get

$$
\begin{aligned}
\left|f(y)-f_{\widetilde{B}(x, r)}\right| & \leq\left|f(y)-f_{\widetilde{B}(y, 2 r)}\right|+\left|f_{\widetilde{B}(y, 2 r)}-f_{\widetilde{B}(x, r)}\right| \\
& \leq C r^{\beta} f_{\beta, 2 r}^{\#}(y)+f_{\widetilde{B}(x, r)}\left|f-f_{\widetilde{B}(y, 2 r)}\right| \\
& \leq C r^{\beta} f_{\beta, 2 r}^{\#}(y) .
\end{aligned}
$$

Now

$$
|f(x)-f(y)| \leq\left|f(x)-f_{\widetilde{B}(x, r)}\right|+\left|f(y)-f_{\widetilde{B}(x, r)}\right|,
$$

and the preceding inequalities lead to

$$
|f(x)-f(y)| \leq C \rho(x, y)^{\beta}\left(f_{\beta, 2 R}^{\#}(x)+f_{\beta, 2 R}^{\#}(y)\right)
$$

for every $x, y \in \Omega_{1} \backslash N$ with $\rho(x, y)<R$. The proof of Lemma 3.3 is complete.

Lemma 3.4. Let $u \in W_{X}^{1, p}(\Omega), 0 \leq \alpha<1$. Then for $R \leq R_{0}$

$$
|u(x)-u(y)| \leq c \rho(x, y)^{1-\alpha}\left(\mathscr{C}_{\alpha, 2 R}|X u|(x)+\mathscr{M}_{\alpha, 2 R}|X u|(y)\right)
$$

for almost every $x, y \in \Omega_{1}$ with $\rho(x, y)<R$. 
Proof. The Poincaré inequality

$$
r^{\alpha-1} f_{\widetilde{B}(x, r)}\left|u-u_{\widetilde{B}(x, r)}\right| \leq c r^{\alpha} f_{\widetilde{B}(x, r)}|X u|,
$$

which holds for all $x \in \Omega_{1}$ and all $r \leq 2 R_{0}$, implies that $u_{1-\alpha, 2 R}^{\#}(x) \leq$ $c \cdot \mathscr{C}_{\alpha, 2 R}|X u|(x)$ for $R \leq R_{0}$. Then Lemma 3.3 gives (27).

Now, Hölder inequality, the decay estimate (25), and the definition of the homogeneous dimension imply that for $\alpha=1-\frac{\gamma}{Q}$ and any $x \in \Omega_{1}$ we have

$$
\mathscr{M}_{\alpha, r_{0} \mid}|X u|(x) \leq\left(\mathscr{M}_{Q \alpha, r_{0}}|X u|^{Q}(x)\right)^{1 / Q} \leq C .
$$

Hence, by Lemma 3.4 we obtain

$$
|u(x)-u(y)| \leq c \rho(x, y)^{\gamma / Q}
$$

for $x, y$ with $\rho(x, y)<r_{0} / 2$. Thus $u$ is locally Hölder continuous with respect to $\rho$ and, by Theorem 2.1, with respect to the Euclidean metric. This concludes the whole proof of Theorem 3.1.

\section{References}

1. Bethuel, F.: On the singular set of stationary harmonic maps. Manuscripta Math. 78 (1993), 417-443.

2. Bethuel, F., Ghidaglia, J.-M.: Improved regularity of solutions to elliptic equations involving Jacobians and applications. J. Math. Pures Appl. 72 (1993), 441-474.

3. Biroli, M. Mosco, U.: Sobolev inequalities on homogeneous spaces. Pot. Anal. 4 (1995), 311 324.

4. Buckley, S., Koskela, P., Lu, G.: Subelliptic Poincare inequalities: the case $p<1$. Publ. Mat. 39 (1995), 313-334.

5. Capogna, L., Danielli, D., Garofalo, N.: An imbedding theorem and the Harnack inequality for nonlinear subelliptic equations. Comm. Partial Diff. Equations 18 (1993), 1765-1794.

6. Capogna, L., Danielli, D., Garofalo, N.: The geometric Sobolev embedding for vector fields and the isoperimetric inequality. Comm. Anal. Geom. 2 (1994), 203-215.

7. Capogna, L., Danielli, D., Garofalo, N.: Capacitary estimates and the local behavior of solutions to nonlinear subelliptic equations. Amer. J. Math. 118 (1996), 1153-1196.

8. Capogna, L., Danielli, D., Garofalo, N.: Subelliptic mollifiers and basic pointwise estimates of Poincaré type, Math. Z. 226 (1997), 147-154.

9. Chanillo, S.: Sobolev inequalities involving divergence-free maps. Comm. P.D.E. 16 (1991), 1969-1994.

10. Chow, W. L.: Über Systeme non linearen partiellen Differentialgleichungen erster Ordnung, Math. Ann. 117 (1939), 98-105.

11. Citti, G.: Semilinear Dirichlet problem involving critical exponents for the Kohn Laplacian. Ann. Mat. Pura Appl. 169 (1995), 375-392.

12. Citti, G., Di Fazio, G.: Hölder continuity of the solutions for operators which are sum of squares of vector fields plus a potential. Proc. Amer. Math. Soc. 122 (1994), 741-750.

13. Citti, G., Garofalo, N., Lanconelli, E.: Harnack's inequality for sum of squares plus potential. Amer. J. Math. 115 (1993), 699-734.

14. Coifman, R., Lions, P. L., Meyer, Y., Semmes, S.: Compensated compactness and Hardy spaces. J. Math. Pures Appl. 72 (1993), 247-286.

15. Coifman, R., Weiss, G.: Analyse harmonique sur certains espaces homogenes, Lecture Notes in Math. 242, Springer 1971. 
16. Danielli, D.: Formulas de representation et théorèmes d'inclusion pour des opérateurs sous elliptiques. C. R. Acad. Sci. Paris 314 (1992), 987-990.

17. Danielli, D., Garofalo, N., Nhieu, D.-M.: Trace inequalities for Carnot-Carathéodory spaces and applications to quasilinear subelliptic equations, (preprint).

18. Evans, L. C.: Partial regularity for stationary harmonic maps into spheres. Arch. Rat. Mech. Anal. 116 (1991), 101-113.

19. Evans, L. C.:, Müller, S.: Hardy spaces and the two-dimensional Euler equations with nonnegative vorticity. J. Amer. Math. Soc. 7 (1994), 199-219.

20. Folland, G. B.: A fundamental solution for a subelliptic operator. Bull. Amer. Math. Soc. 79 (1973), 373-376.

21. Folland, G. B.: Subelliptic estimates and function spaces on nilpotent Lie groups. Arkiv för Mat. 13 (1975), 161-207.

22. Folland, G. B., Stein, E. M.: Hardy spaces on homogeneous groups. Mathematical Notes vol. 28, Princeton Univ. Press, Princeton, New Jersey, 1982.

23. Franchi, B.: Weighted Sobolev-Poincaré inequalities and pointwise estimates for a class of degenerate elliptic operators. Trans. Amer. Math. Soc. 327 (1991), 125-158.

24. Franchi, B., Gallot, S., Wheeden, R. L.: Sobolev and isoperimetric inequalities for degenerate metrics. Math. Ann. 300 (1994), 557-571.

25. Franchi, B., Gutiérrez C., Wheeden, R. L.: Weighted Poincaré-Sobolev inequalities for inequalities for Grushin type operators. Comm. Partial Diff. Equations 19 (1994), 523-604.

26. Franchi, B. and Lanconelli, E.: Hölder regularity theorem for a class of non uniformly elliptic operators with measurable coefficients, Ann. Scuola Norm. Sup. Pisa 10 (1983), 523-541.

27. Franchi, B., Lu, G., Wheeden, R. L.: Representation formulas and weighted Poincaré inequalities for Hörmander vector fields. Ann. Inst. Fourier (Grenoble) 45 (1995), 577-604.

28. Franchi, B., Lu, G., Wheeden, R. L.: A relationship between Poincaré type inequalities and representation formulas in spaces of homogeneous type. Int. Mat. Res. Notices (1996), No. 1, $1-14$.

29. Franchi, B., Serapioni, R., Serra Cassano, F.: Approximation and imbedding theorems for weighted Sobolev spaces associated with Lipschitz continuous vector fields. Bolletino U.M.I. 11-B (1997), 83-117.

30. Friedrichs, K. O.: The identity of weak and strong extensions of differential operators. Trans. Amer. Math. Soc. 55 (1944), 132-151.

31. Fuchs, M.: The blow-up of $p$-harmonic maps. Manuscripta Math. 81 (1993), 89-94.

32. Garofalo, N., Lanconelli, E.: Existence and nonexistence results for semilinear equations on the Heisenberg group. Indiana Univ. Math. J. 41 (1992), 71-98.

33. Garofalo, N., Nhieu, D. M.: Lipschitz continuity, global smooth approximation and extension theorems for Sobolev functions in Carnot-Carathéodory spaces, (preprint).

34. Garofalo, N., Nhieu, D. M.: Isoperimetric and Sobolev inequalities for Carnot-Carathéodory spaces and the existence of minimal surfaces, Comm. Pure Appl. Math. 49 (1996), 1081-1144.

35. Grigor'yan, A.: The heat equation on noncompact Riemannian manifolds. Mat. Sb. 182, (1991), 55-87 (Russian).

36. Gromov, M.: Carnot-Carathéodory spaces seen from within, in: Sub-Riemannian Geometry, pp. 85-318; Progress in Math. vol. 144 (A. Bellaïche, J.-J. Risler), Birkhäuser, 1996.

37. Hajlasz, P., Kinnunen, J.: Hölder quasicontinuity of Sobolev functions on metric spaces, to appear in Revista Math. Iberoamericana.

38. Hajlasz, P., Koskela, P.: Sobolev meets Poincaré, C. R. Acad. Sci. Paris 320 (1995), 1211-1215.

39. Hajlasz, P., Koskela, P.: Sobolev met Poincaré, preprint.

40. Hélein, F.: Regularité des applications faiblement harmoniques entre une surface et une sphère. C. R. Acad. Sci. Paris 311 (1990), 519-524.

41. Hélein, F.: Régularité des applications faiblement harmoniques entre une surface et une variété riemannienne. C. R. Acad. Sci. Paris 312 (1991), 591-596.

42. Hélein, F.: Harmonic maps, conservation laws and moving frames. Editions Frontieres, Diderot Publishers, Paris 1997.

43. Jerison, D.: The Poincaré inequality for vector fields satisfying Hörmander's condition, Duke Math. J. 53 (1986) 503-523.

44. Jerison, D., Lee, J. M.: Intrinsic CR coordinates and the CR Yamabe problem. J. Diff. Geom. 29 (1989), 303-343 
45. Jerison, D., Lee, J. M.: Extremals for the Sobolev inequality on the Heisenberg group and the CR Yambe problem. J. Amer. Math. Soc. 1 (1988), 1-13.

46. Jost, J.: Generalized harmonic maps between metric spaces, in: Geometric analysis and the calculus of variations (J. Jost, ed.), International Press, 1966, pp. 143-174.

47. Jost, J.: Generalized Dirichlet forms and harmonic maps. Calc. Var. 5 (1997), 1-19.

48. Jost, J., Xu, C. J.: Subelliptic harmonic maps, to appear in Trans. Amer. Math. Soc.

49. Kaplan, A.: Fundamental solution for a class of hypoelliptic PDE generated by composition of quadratic forms. Trans. Amer. Math. Soc. 258 (1980), 147-153.

50. Lu, G.: Weighted Poincaré and Sobolev inequalities for vector fields satisfying Hörmander's condition and applications. Rev. Mat. Iberoamericana, 8 (1992), 367-439.

51. Lu, G.: The sharp Poincaré inequality for free vector fields: An endpoint result, Revista Mat. Iberoamericana, 10 (1994), 453-466.

52. Lu, G.: Embedding theorems on Campanato-Morrey spaces for vector fields and applications. C. R. Acad. Sci. Paris. 320 (1995), 429-434.

53. Lu, G.: Embedding theorems into Lipschitz and BMO spaces and applications to quasilinear subelliptic differential equations. Publ. Mat. 40 (1996), 301-329.

54. Lu, G.: A note on a Poincare type inequality for solutions to subelliptic equations. Comm. Partial Diff. Equations 21 (1996), 235-254.

55. Macías, R. A., Segovia, C.: A decomposition into atoms of ditributions on spaces of homogeneous type. Adv. Math. 33 (1979), 271-309.

56. Maheux, P., Saloff-Coste, L.: Analyse sur les boules d'un opérateur sous-elliptique, Math. Ann. 303 (1995), 713-740.

57. Mitchell, J.: On Carnot-Carathéodory metrics, J. Diff. Geom. 21 (1985), 35-45.

58. Mou, L., Yang, P.: Regularity for $n$-harmonic maps, J. Geom. Anal. 6 (1996), 91-112.

59. Müller, S.: Higher integrability of determinants and weak convergence in $L^{1}$. J. Reine Angew. Math. 412 (1990), 20-34.

60. Müller, S., Šverák, V.: On surfaces of finite total curvature. J. Diff. Geom. 42 (1995), 229-258.

61. Nagel, A., Stein, E. M. and Wainger, S.: Balls and metrics defined by vector fields I: Basic properties, Acta Math. 155 (1985), 103-147.

62. Rothschild, L. P., Stein, E. M.: Hypoelliptic differential operators and nilpotent groups. Acta Math. 137 (1976), 247-320.

63. Saloff-Coste, L.: A note on Poincaré, Sobolev, and Harnack inequalities, Internat. Mat. Res. Notices 1992 no. 2, pp. 27-38

64. Sánchez-Calle, A.: Fundamental solutions and geometry of sums of squares of vector fields, Invent. Math. 78 (1984), 143-160.

65. Semmes, S.: A primer on Hardy spaces and some remarks on a theorem of Evans and Müller. Comm. P.D.E. 19 (1994), 277-319.

66. Stein, E.: Harmonic Analysis: Real-Variable Methods, Orthogonality and Oscillatory Integrals, Princeton Univ. Press 1993.

67. Strzelecki, P.: Regularity of $p$-harmonic maps from $p$-dimensional ball into a sphere. Manuscr. Math. 82 (1994), 407-415.

68. Strzelecki, P.: Stationary $p$-harmonic maps into spheres. Banach Center Publ. vol. 33, pp. 381391, Warszawa, 1996

69. Sturm, K. T.: Analysis on local Dirichlet spaces III. The parabolic Harnack inequality. J. Math. Pures Appl. 75 (1996), 273-297.

70. Takeuchi, H.: Some conformal properties of $p$-harmonic maps and a regularity for sphere valued $p$-harmonic maps, J. Math. Soc. Japan 26 (1994), 217-334.

71. Toro, T., Wang, C.: Compactness properties of weakly $p$-harmonic maps into homogeneous spaces, Indiana Univ. Math. J. 44 (1995), 87-113.

72. Varopoulos, T.: Functions harmoniques sur les groupes de Lie, C. R. Acad. Sci. Paris 304 (1987), 519-521.

73. Varopoulos, T., Saloff-Coste, L., and Coulhon, T.:, Analysis and geometry on groups, Cambridge Univ. Press 1992

74. Vodop'yanov, S. K.: Weighted Sobolev spaces and the boundary behavior of solutions of degenerate hypoelliptic equations. Sibirsk. Mat. Zh. 36 (1995), 278-300, (Russian), English translation: Siberian Math. J.: 36 (1995), 246-264.

75. Vodop'yanov, S. K., Chernikov.: Sobolev spaces and hypoelliptic equations, to appear in Siberian 
Adv. Math.

76. Vodop'yanov, S. K., Markina, I. G.: Exceptional sets for solutions of subelliptic equations. Sibirsk. Mat. Zh. 36 (1995), 805-818, (Russian), English translation: Siberian Math. J. 36 (1995), 694-706.

77. Xu, C. J.: Subelliptic variational problems. Bull. Soc. Math. France 118 (1990), 147-159.

78. Xu, C. J.: Regularity for quasilinear second-order subelliptic equations. Comm. Pure Appl. Math. 45 (1992), 77-96. 